\title{
Combined chemical and structural analysis of low dimensional systems in FEG-SEM
}

Purvesh Soni ${ }^{1}$, Laurie Palasse ${ }^{2}$ and Meiken Falke ${ }^{1}$

${ }^{1}$ Bruker Nano GmbH, United States, ${ }^{2}$ Bruker Nano Analytics, Berlin, Berlin, Germany

Correlative microscopy is a powerful analytical approach combining strengths of different techniques for study of materials. In this study we present simultaneous chemical and structural analysis of FIB-lamella extracted from $\mathrm{Cu}(\mathrm{In}, \mathrm{Ga}) \mathrm{Se} 2$ (CIGS) solar cells in SEM.

Chemical analysis of low-dimensional systems (nanoparticles, nanorods, TEM lamellae, etc.) in SEM is highly desirable considering the lower ownership and operational costs, ease of use, and dual-beam capabilities for sample preparation. Due to geometric and other constrains, the solid angle which can be achieved for conventional EDS detectors in SEM, is on average a magnitude lower compared to TEM. Moreover, the small interaction volume of electron transparent materials generates lower $\mathrm{x}$-ray yield than bulk, which complicates reliable chemical identification. All this makes EDS analysis of e-transparent samples in SEM challenging.

For this work, we used an annular side entry detector with a central aperture, the XFlash®FlatQUAD EDS detector (Fig. 1) [1,2]. Unlike the conventional EDS detectors placed on high angle ports, the FlatQUAD is inserted horizontally and placed between the sample and the pole piece. Due to the unique detector position, a high solid angle ( 1 steradian) for $\mathrm{x}$-ray collection is achieved giving high sensitivity at low count rates. Furthermore, the sample-detector geometry enables a large take-off angle, allowing the analysis of highly topographic or bent specimens.

Simultaneous EDS and transmission Kikuchi diffraction (TKD) measurements are possible due to the unique sample-detector geometry. The sample is placed between the FlatQUAD and OPTIMUS TKD detector [3]. As shown in figure 1, TKD and EDS signals are simultaneously collected by both detectors. Since the OPTIMUS TKD detector and the specimen are aligned with the primary e-beam "on-axis" [4], TKD patterns are collected with minimal band distortions and fast measurement speeds at low probe currents. Since the Boersch effect is minimized, higher spatial resolution can be achieved.

Figure 2a shows single element EDS maps of the CIGS absorber and CdS buffer layer. For electrontransparent samples, the Cliff-Lorimer-factor or Zeta-factor based quantification methods must be used [5,6]. Here we use the Cliff-Lorimer method to quantify the element distribution and the line profile (Fig. 2b) for CIGS solar cells. Figure 2c presents TKD results from four different layers of a CIGS solar cell. The map has a high indexing rate of nearly $100 \%$ in the CIGS region. The thin CdS layer (between $\mathrm{ZnO}$ and CIGS) could not be measured because the very fineCdS particles $(<10 \mathrm{~nm})$ produce TKD pattern overlaps. However, this is a limitation due to sample thickness and can be resolved by using a thinner sample ( 20-30 nm).

The successful combination of quantitative element and orientation mapping demonstrates how new instrumentation enables the correlation of different analysis techniques in SEM. Even more correlative research, e.g. the addition of $\mu-X R F$, WDS and in-situ experiments in SEM is possible. 


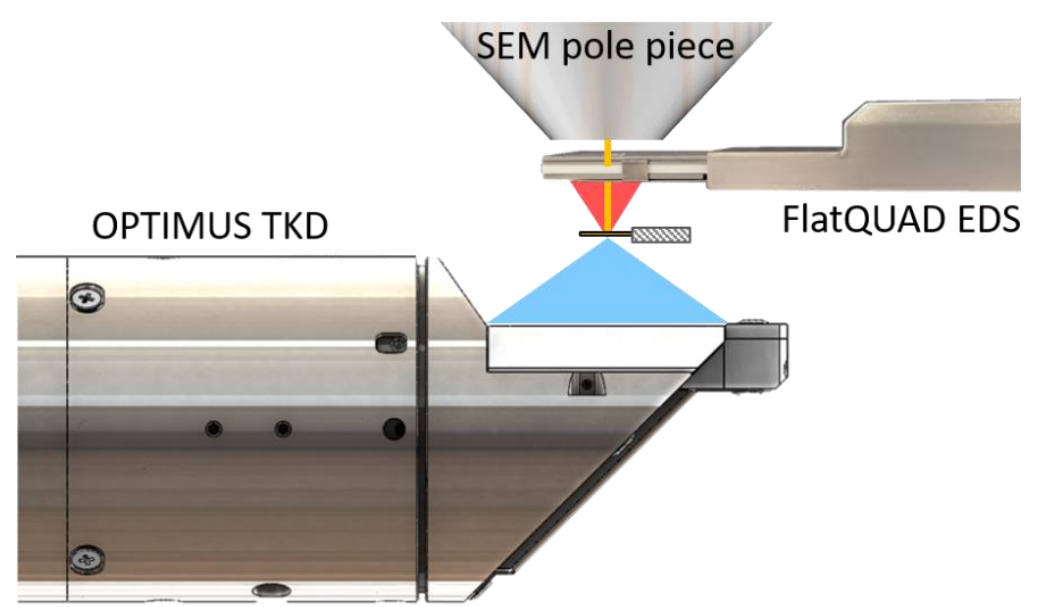

Chemical information

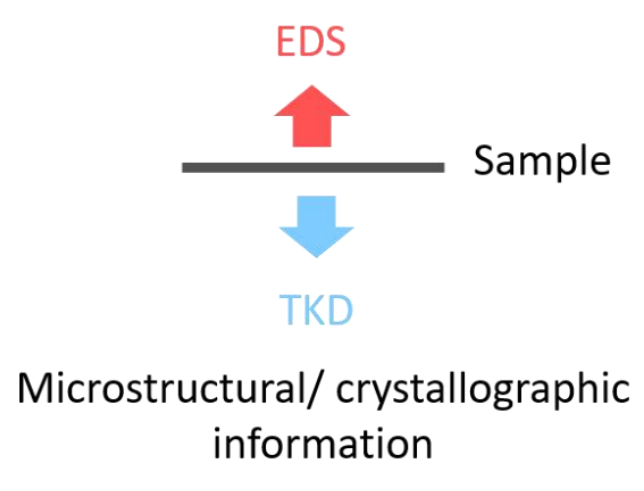

Figure 1. Sample-detector geometry showing simultaneous use of the annular FlatQUAD EDS and OPTIMUS TKD detectors for e-transparent samples.

(a)

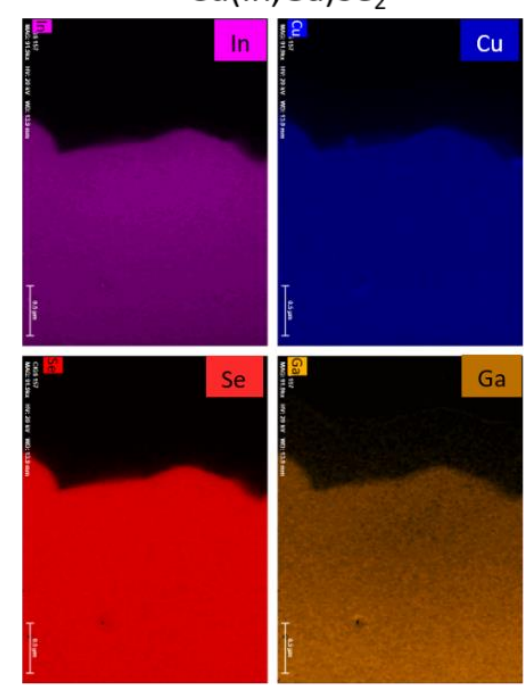

(b) (c)
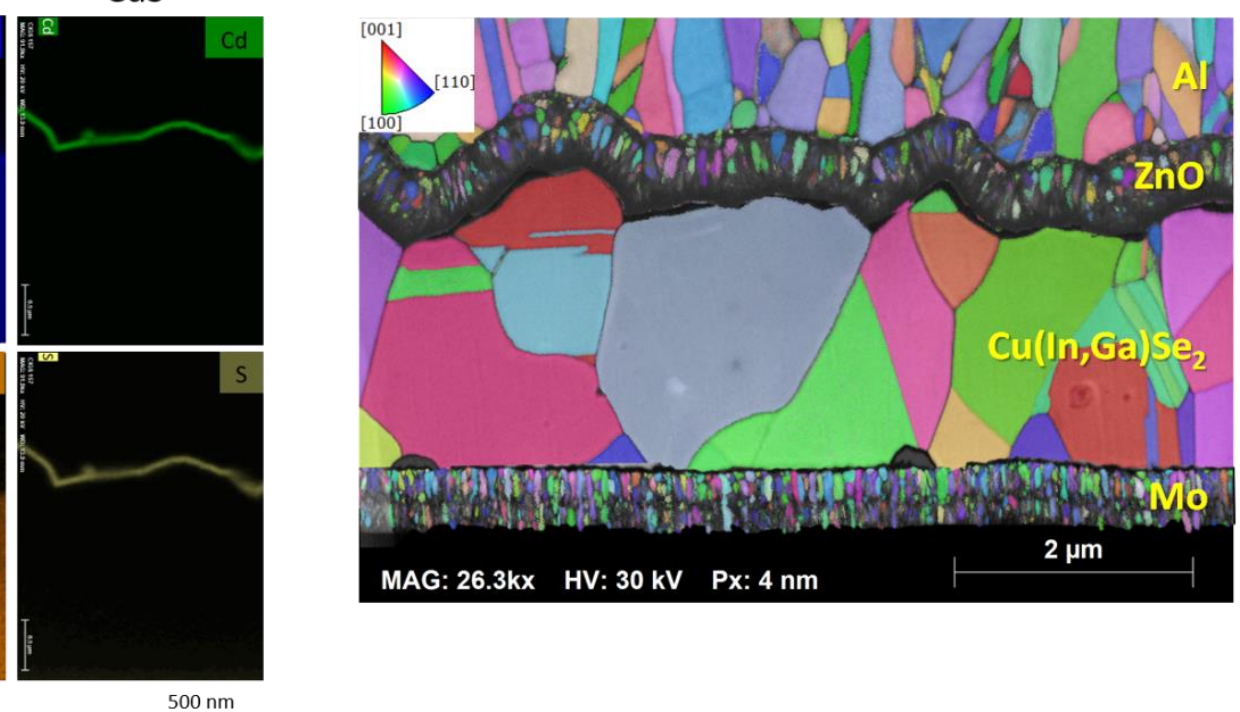
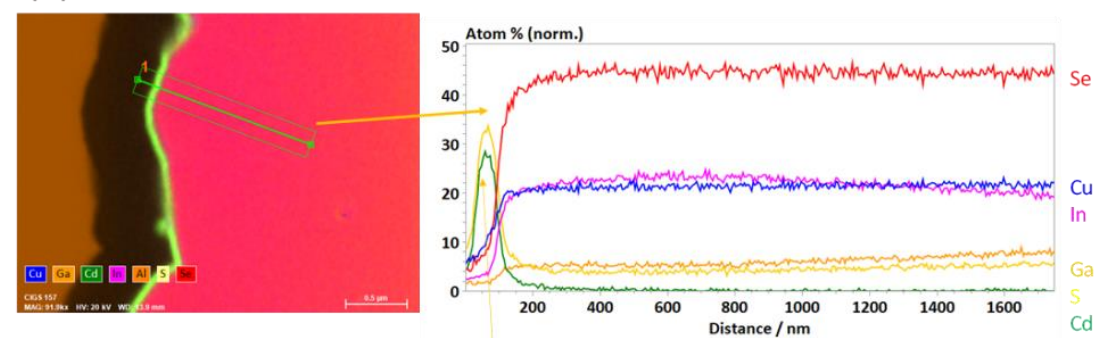

Figure 2. Single element EDS maps (a) of CIGS absorber and CdS buffer layer, with the corresponding quantified line profile (b) using the Cliff-Lorimer quantification method. TKD map (c) showing the crystal orientation distribution in Z-direction (perpendicular to the lamella surface) acquired from four different layers of a CIGS solar cell. 


\section{References}

[1] Kotula, P., Michael, J., \& Rohde, M. (2008). Results from Two Four-Channel Si-drift Detectors on an SEM: Conventional and Annular Geometries. Microscopy and Microanalysis, 14(S2), 116-117. doi:10.1017/S1431927608081701

[2] Terborg R., Rohde M. (2008) New developments in state of the art silicon drift detectors (SDD) and multiple element SDD. In: Luysberg M., Tillmann K., Weirich T. (eds) EMC 2008 14th European Microscopy Congress 1-5 September 2008, Aachen, Germany. Springer, Berlin, Heidelberg. https://doi.org/10.1007/978-3-540-85156-1_317

[3] KELLER, R. and GEISS, R. (2012), Transmission EBSD from $10 \mathrm{~nm}$ domains in a scanning electron microscope. Journal of Microscopy, 245: 245-251. https://doi.org/10.1111/j.1365-2818.2011.03566.x

[4] Brodu, E., Bouzy, E., \&Fundenberger, J. (2017). On-axis Transmission Kikuchi Diffraction for Orientation Mapping of Nanocrystalline Materials in the SEM. Microscopy and Microanalysis, 23(S1), 530-531. doi:10.1017/S1431927617003336

[5] Kothleitner, G., Grogger, W., Dienstleder, M., \& Hofer, F. (2014). Linking TEM Analytical Spectroscopies for an Assumptionless Compositional Analysis. Microscopy and Microanalysis, 20(3), 678-686. doi:10.1017/S1431927614000130

[6] Watanabe M, Williams DB. The quantitative analysis of thin specimens: a review of progress from the Cliff-Lorimer to the new zeta-factor methods. J Microsc. 2006 Feb;221(Pt 2):89-109. doi: 10.1111/j.1365-2818.2006.01549.x. PMID: 16499549. 